\title{
NR2B-Containing Receptors Mediate Cross Talk among Hippocampal Synapses
}

\author{
Annalisa Scimemi, ${ }^{1}$ Alan Fine, ${ }^{2}$ Dimitri M. Kullmann, ${ }^{1}$ and Dmitri A. Rusakov ${ }^{1}$ \\ ${ }^{1}$ Institute of Neurology, University College London, London WC1N 3BG, United Kingdom, and 2Division of Neurophysiology, National Institute for Medical \\ Research, London NW7 1AA, United Kingdom
}

\begin{abstract}
Under some conditions, synaptically released glutamate can exert long-range actions in the cortical microcircuitry. To what extent glutamate spillover leads to direct cross talk among individual synapses remains unclear. We recorded NMDAR-mediated EPSCs in acute hippocampal slices at $35^{\circ} \mathrm{C}$ by stimulating two independent pathways that converge on the same CA1 pyramidal cell. Activation of a conditioning pathway in the presence of the use-dependent blocker dizocilpine maleate (MK801) resulted in partial NMDA receptor (NMDAR) blockade in the other, silent pathway. This was accompanied by an increase in the rise time of the EPSCs in the conditioning (although not the silent) pathway, implying an increase in diffusional distance from release site to NMDARs. We estimated that up to $\sim 30 \%$ of NMDARs contributing to EPSCs were activated by glutamate released from multiple synaptic sources; however, NMDARmediated synaptic cross talk was undetectable when NR2B subunit-containing receptors were blocked (but could be rescued by blocking glutamate uptake). We propose that NR2B-containing NMDARs can detect glutamate arising from multiple synapses, whereas NR2Acontaining NMDARs only normally mediate direct synaptic transmission. These NMDAR isoforms thus play complementary roles in sensing global and local glutamate signals, respectively.
\end{abstract}

Key words: hippocampus; glutamate; spillover; NMDA receptors; NR2B; synapse

\section{Introduction}

Recent evidence suggests that synaptically released glutamate not only acts locally at intrasynaptic receptors, but it also escapes from the synaptic cleft to exert remote actions. Glutamate spillover, for instance, modulates GABA release in the cerebellum (Mitchell and Silver, 2000; Satake et al., 2000) and hippocampus (Min et al., 1998; Semyanov and Kullmann, 2000; Cossart et al., 2001; Semyanov and Kullmann, 2001). Repeated activation of cerebellar parallel fibers not only activates extrasynaptic NMDA receptors (NMDARs) (Clark and Cull-Candy, 2002) but also receptors on interneurons that are relatively far from the active synapses (Carter and Regehr, 2000). In the hippocampus, glutamate escape is implicated in the activation of extrasynaptic glutamate receptors and in heterosynaptic modulation of mossy fiber transmission (Vogt and Nicoll, 1999; Schmitz et al., 2001).

It is much less clear to what extent direct NMDAR-mediated cross talk occurs among small excitatory synapses (Kullmann, 1994; Asztely et al., 1997; Franks et al., 2002; Lehre and Rusakov, 2002). Diamond (2001) showed recently that a rapidly dissociating competitive NMDAR antagonist selectively reduced the slow component of NMDAR EPSCs. Although this implies that glutamate persists for a long time in the extracellular space and may

\footnotetext{
Received Feb. 2, 2004; revised April 6, 2004; accepted April 6, 2004.

This work was supported by the Medical Research Council, the Wellcome Trust, and the European Commission (DECG QLG3-CT-2001-02004). We are grateful to Drs. L. Trussell, T. V. P. Bliss, N. J. Emptage, D. Attwell, C. Reid, and M. Walker for helpful comments.

Correspondence should be addressed to D. A. Rusakov, Institute of Neurology, University College London, Queen Square, London WC1N 3BG, UK. E-mail: d.rusakov@ion.ucl.ac.uk.

DOI:10.1523/JNEUROSCI.0364-04.2004

Copyright $\odot 2004$ Society for Neuroscience $\quad$ 0270-6474/04/244767-11\$15.00/0
}

activate extrasynaptic NMDARs, it does not necessarily follow that cooperation between neighboring synapses normally occurs. Arnth-Jensen et al. (2002) showed that increasing the density of release sites prolongs the decay of NMDAR EPSCs. Because responses of independent synapses were assumed to summate linearly, the increased decay suggested synaptic cooperation; however, because the extracellular space in CA1 hosts on average $\sim 0.2 \mathrm{~mm}$ high-affinity glutamate transporters (Lehre and Danbolt, 1998), glutamate originating from multiple sources could summate supralinearly in the extracellular space (see Materials and Methods). This suggests a scenario in which glutamate released from any individual site does not reach NMDARs in a recorded cell, whereas synchronous release from multiple sites partly overcomes the transporters, leading to activation of remote NMDARs. Because these receptors contribute to the slow current component, the observations of Arnth-Jensen et al. (2002) do not necessarily indicate that direct synaptic cross talk normally occurs.

Here we use an alternative approach to estimate the degree of NMDAR-mediated cross talk between two separately activated pathways. We also ask how different NMDAR subtypes contribute to this phenomenon. We find that, at a near-physiological temperature, up to $30-35 \%$ of NMDARs (depending on the density of active synapses and the efficiency of glutamate uptake) can be activated by more than one synaptic release site. Surprisingly, blockade of NR2B-containing NMDARs completely abolishes this intersynaptic cross talk, although it can be rescued by blocking glutamate uptake. The results argue for complementary roles of NR2A- and NR2B-containing NMDARs in sensing local versus global glutamate release in the cortex. 


\section{Materials and Methods}

Male Sprague Dawley rats, 4-8 weeks old, were killed by cervical dislocation followed by decapitation. Hippocampal slices (350 $\mu \mathrm{m}$ thick) were transferred to a submersion-type recording chamber. The perfusion medium contained (in $\mathrm{mm}$ ): $119 \mathrm{NaCl}, 2.5 \mathrm{KCl}, 1 \mathrm{MgCl}_{2}, 2 \mathrm{CaCl}_{2}, 26.2$ $\mathrm{NaHCO}_{3}, 1 \mathrm{NaH}_{2} \mathrm{PO}_{4}, 22$ glucose, and 0.1 picrotoxin, and was bubbled with $95 \% \mathrm{O}_{2} / 5 \% \mathrm{CO}_{2}$. All experiments were performed at $35-36^{\circ} \mathrm{C}$. Stimuli (100 $\mu$ sec square pulses) were delivered via bipolar stainless steel electrodes positioned in stratum radiatum. Whole-cell recordings were made with patch pipettes containing (in mM): 117.5 Cs gluconate, 17.5 CsCl, 10 HEPES, 0.2 EGTA, $8 \mathrm{NaCl}, 2 \mathrm{Mg}$-ATP, 0.3 GTP, and 5 QX314 $\mathrm{Br}, \mathrm{pH}$ 7.2, $290 \mathrm{mOsm}$. The series resistance was monitored throughout the experiment using a $-3 \mathrm{mV}$ step command and was in the range of 7-14 $\mathrm{M} \Omega$; cells showing an unstable ( $>30 \%$ change) series resistance or holding current were rejected. Evoked NMDAR EPSCs were recorded at $V_{\mathrm{m}}=+40$ $\mathrm{mV}$ in $25 \mu \mathrm{M}$ 2,3-dioxo-6-nitro-1,2,3,4-tetrahydrobenzo[f] quinoxaline-7sulfonamide disodium, collected in series of $20-50$ trials ( $>5 \mathrm{sec}$ apart) of $150-500$ msec duration using a $2 \mathrm{kHz}$ low-pass filter (Axopatch 200B, Axon Instruments, Foster City, CA), and analyzed off-line (LabView, National Instruments); the intertrial interval and the number of trials in the presence of dizocilpine maleate (MK801) were set so that the total exposure to MK801 was similar ( $\sim 15$ or $30 \mathrm{~min})$ in all experiments in which the matching was required. Five minutes were allowed for wash-in and washout of MK801. NMDAR EPSC amplitudes were measured as the average current over a 100 msec interval after onset; this measure was selected to reduce the noiserelated error of individual EPSC measurements. Application of MK801 reduced substantially the decay time constant of EPSCs, consistent with a reduction in the average NMDAR open time (Jahr, 1992; Rosenmund et al., 1993). This effect was only partly reversed after 5 min washout of MK801, indicating that washout was incomplete. To reduce possible bias caused by incomplete washout, we used only the first eight responses recorded after restarting stimulation. The amplitude variability of these responses was comparable with that of baseline responses: SEM $<3-5 \%$ of the average amplitude. [This differed slightly from the approach of Carter and Regehr (2000), who measured a single response without washing out MK801.] The EPSC latency was measured at $70 \%$ of peak, which coincides roughly with the end of a near-linear segment in the onset of the average EPSC. The shortening of the EPSC rise time by MK801 (Rosenmund et al., 1993) was undetectable, probably because it was masked by reduced local shunting as the synaptic conductance decreased (see below). Chemicals were purchased from Sigma (St. Louis, MO), except for receptor antagonists and DL-threo$\beta$-benzyloxyaspartate (TBOA), which were purchased from Tocris Cookson.

Supralinear summation of remotely activated NMDARs: kinetic estimates. To assess whether remote glutamate transients scale up linearly in the presence of glutamate transporters, we first used a simple approximation. Because the wave of glutamate released from a remote site can be considered quasi steady state within a few milliseconds of release (at least on a millisecond scale of changes), the kinetics of reversible binding to transporters leads to a quadratic equation that relates the concentrations of total and free glutamate, $G_{\text {tot }}$ and $G$ :

$$
G^{2}+G\left(T_{\text {tot }}-G_{\text {tot }}+K_{\mathrm{d}}\right)-K_{\mathrm{d}} G_{\text {tot }}=0,
$$

where $T_{\text {tot }}(\sim 200 \mu \mathrm{M})$ and $K_{\mathrm{d}}(\sim 20 \mu \mathrm{M})$ stand for the known total extracellular concentration of (glial) transporters and their effective dissociation constant, respectively, in the CA1 neuropil (Danbolt, 2001). The NMDAR kinetics implies significant activation when glutamate is in the micromolar range (Lester and Jahr, 1992). It then follows from Equation 1 that, to reach $G=2.0 \mu \mathrm{m}$ in the extracellular space, the total glutamate should be in the region of $G_{\text {tot }}=20 \mu \mathrm{M}$; this is also consistent with a detailed model of glutamate release and diffusion (Lehre and Rusakov, 2002). In these conditions, a fourfold increase in the number of release sites can be represented by substituting $G_{\text {tot }}=4 \times 20 \mu \mathrm{M}=80 \mu \mathrm{M}$ in Equation 1, which then yields $G=10.6 \mu \mathrm{m}$. This implies that the free glutamate transient increases $33 \%$ above what is expected from linear summation, to $10.6 \mu \mathrm{M}$ instead of the expected $4 \times 2.0 \mu \mathrm{M}=8.0 \mu \mathrm{M}$. Addressing the same question with a more explicit modeling experiment (see below), in which glutamate transients generated at $0.5-1.0 \mu \mathrm{m}$ from the release sites are added in the presence of glutamate transporters, gives qualitatively similar results (data not shown).

Perisynaptic diffusion of glutamate. To simulate release, diffusion, and uptake of glutamate in the synaptic vicinity, we represented a "typical" CA1 excitatory synapse and its microenvironment with a threedimensional (3D) compartmental model (with space partitioned both radially and tangentially) (Rusakov, 2001; Lehre and Rusakov, 2002). Briefly, the synaptic geometry included a flat cleft (width, $20 \mathrm{~nm}$ ) between two hemispheres (consistent obstacles to diffusion; radius, 0.11 $\mu \mathrm{m}$ ) separated from a homogeneous porous medium by a $20 \mathrm{~nm}$ gap. The perisynaptic medium had an extracellular fraction $\alpha=0.12$, tortuosity $\lambda=1.4$, and a diffusion coefficient in the interstitial space $D=0.4$ $\mu \mathrm{m}^{2} / \mathrm{msec}$. This value of $D$ is the upper limit estimate based on direct physical measurements of ion diffusivity in a narrow extracellular cleft (Kiessling et al., 2000). Compared with previous models (Rusakov and Kullmann, 1998; Barbour, 2001), this approach avoided the simplifying limitations of spherical symmetry and could incorporate glutamate transporters unevenly, in accordance with quantitative electron microscopy data (Lehre and Rusakov, 2002). Glutamate binding and uptake were represented by a simplified kinetic scheme (reflecting the upper limit of uptake and therefore a conservative estimate for extrasynaptic glutamate escape):

$$
G l u+T \underset{k_{-1}}{\stackrel{k_{1}}{\longleftrightarrow}} G l u T \stackrel{k_{2}}{\longrightarrow} G l u_{\mathrm{in}}+T,
$$

where $G l u, T$, and GluT indicate free glutamate, free transporter, and the glutamate-transporter complex, respectively, $k_{1}=10^{4} \mathrm{M}^{-1} / \mathrm{msec}^{-1}$, and $k_{-1}=0.2 \mathrm{msec}^{-1}$; and the cycling rate set at its upper limit at $36^{\circ} \mathrm{C}, k_{2}=$ $0.1 \mathrm{msec}^{-1}$ (Bergles and Jahr, 1998). This kinetics was translated into a set of finite-difference equations computed for each space compartment [note typographical errors in Eq. 3 (Rusakov, 2001)]:

$$
[G l u]_{\mathrm{t}}=[G l u]_{\mathrm{t}-\mathrm{dt}}+\left(-k_{1}[G l u]_{\mathrm{t}-\mathrm{dt}}[T]_{\mathrm{t}-\mathrm{dt}}+k_{-1}[G l u T]_{\mathrm{t}-\mathrm{dt}}\right) d t,
$$

$$
\begin{aligned}
{[G l u T]_{\mathrm{t}}=[G l u T]_{\mathrm{t}-\mathrm{dt}}+\left\{-\left(k_{-1}+k_{2}\right)[G l u T]_{\mathrm{t}-\mathrm{dt}}\right.} & \\
& \left.+k_{1}[G l u]_{\mathrm{t}-\mathrm{dt}}[T]_{\mathrm{t}-\mathrm{dt}}\right\} d t, \\
& {[G l u T]_{\mathrm{t}}+[T]_{\mathrm{t}}=[G l u T]_{\mathrm{t}-\mathrm{dt}}+[T]_{\mathrm{t}-\mathrm{dt}}=\left[T_{\mathrm{tot}}\right], }
\end{aligned}
$$

where the brackets indicate concentrations, time points are denoted by subscripts, and $\left[T_{\text {tot }}\right]$ is the total extracellular concentration of transporters.

At each given space compartment, the NMDAR current was computed from the diffusion profiles of glutamate and a multistep NMDAR kinetic scheme (Lester and Jahr, 1992), as described previously (Rusakov and Kullmann, 1998). Because the extracellular concentration of highaffinity glutamate transporters is much higher than that of ionotropic glutamate receptors (Lehre and Danbolt, 1998; Nusser et al., 1998; Takumi et al., 1999; Racca et al., 2000), the effect of NMDARs on the glutamate concentration profile was considered negligible compared with that of transporters. Within the synaptic cleft, which is devoid of glial transporters, the same logic applies, simply because the total number of available NMDARs $(20-40)$ is small compared with the number of released glutamate molecules (3000-5000). The EPSC latency changes (see Fig. $4 C, D$ ) were assessed using a single synapse model, with a cutoff distance of $5 \mu \mathrm{m}$.

To compute the average glutamate transient after synchronous release from several synapses, we adopted a conservative estimate of glutamate spillover. Spatial profiles of glutamate released from individual synapses scattered randomly within a $5-\mu \mathrm{m}$-sided cube were summated, and possible supralinear summation (attributable to the lower glutamate-transporter ratio after single, as opposed to multiple, synaptic release; see Supralinear summation above) was ignored. For simplicity, and to account for the arbitrary choice of presynaptic and postsynaptic sides at each synapse, glutamate concentrations at different distances from individual release sites were computed for space compartments coplanar to 
A

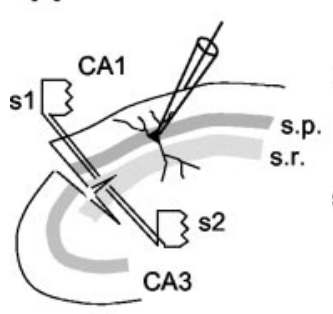

B

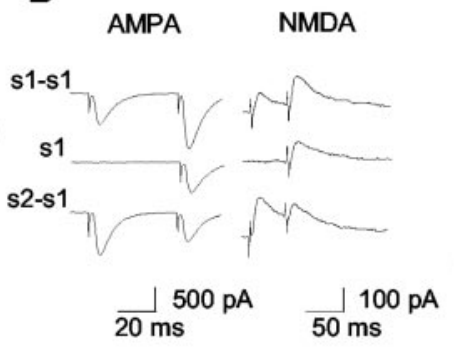

C

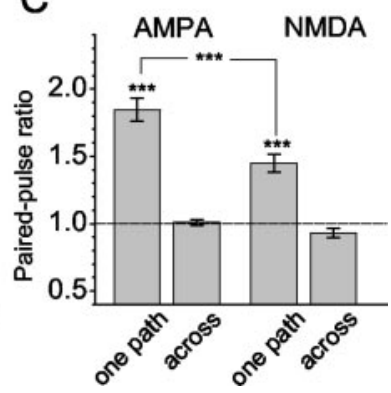

Figure 1. Stimulating two independent pathways converging on a CA1 pyramidal cell. $A$, Schematic illustration of experimental design: stratum radiatum (s.r.) was divided by a cut parallel to stratum pyramidale (s.p.), and a bipolar stimulating electrode was placed in each half to activate two pathways ( 1 and s2); EPSC $s$ were recorded in CA1 pyramidal cells in whole-cell mode. $B$, The amplitude ratio between the second and first responses (paired-pulse ratio) reveals PPF within each pathway but shows no evidence of cross PPF between the two. Example traces (average of 30; left and right panels, AMPAR and NMDAR EPSCs, respectively); s1 and s2, stimulation of pathway 1 or 2. C, Statistical summary of PPF values for AMPAR $(n=16)$ and $\operatorname{NMDAR}(n=18) \operatorname{EPSC}$.

the synaptic cleft. The number of molecules released from an individual site was set at 5000 . This value may be in excess of the glutamate contents of an individual vesicle with dimensions estimated from a recent ultrastructural study (Schikorski and Stevens, 1997), although it is consistent with other estimates (Harris and Sultan, 1995), including one based on rapid freeze-fracture and negative-staining electron microscopy (Zampighi and Fisher, 1997). Moreover, it represents a conservative estimate of the amount of glutamate exocytosed if multivesicular release occurs (Oertner et al., 2002). The total number of modeled synapses (250) matched the experimental synaptic density in area CA1 of 2.0 $\mu \mathrm{m}^{-3}$ (Rusakov et al., 1998). "Active" synapses in the scatter were selected randomly within the 5 - $\mu \mathrm{m}$-wide cube (with a $1 \mu \mathrm{m}$ minussampling margin).

Synaptic current shunt. In the whole-cell configuration, the simplified recording circuit includes a pipette access resistance $R_{\text {pip }}$, an equivalent cell membrane resistance $R_{\mathrm{m}}$ and capacitance $C_{\mathrm{m}}$, and a variable synaptic resistance $R_{\text {syn }}$. When the voltage is clamped, the total circuit impedance $Z_{\text {tot }}$ at the input of the amplifier is $Z_{\text {tot }}=R_{\text {pip }}+\left(R_{\text {syn }}{ }^{-1}+R_{\mathrm{m}}{ }^{-1}+\right.$ $\left.X_{\mathrm{C}}{ }^{-1}\right)^{-1}$, where $X_{\mathrm{C}}=\omega C_{\mathrm{m}}$ is the reactance of the capacitor $C_{\mathrm{m}}$ at a cycling frequency $\omega$. The typical parameters of this circuit in the conditions of our experiments are as follows: (1) in the resting conditions, $X_{\mathrm{C}}$ $=\infty, R_{\text {syn }}=\infty$, and $R_{\text {tot }}$ is $300-500 \mathrm{M} \Omega$, (2) the pipette resistance is $\sim 2.5$ $\mathrm{M} \Omega$, and (3) a $5 \mathrm{mV}$ command voltage step (in the absence of synaptic activation, $R_{\text {syn }}=\infty$ ) shows $Z_{\text {tot }}$ as an equivalent series resistance of $<10-12 \mathrm{M} \Omega$ for the fastest current components and $\sim 20 \mathrm{M} \Omega$ for current components with a rise time constant of $8-15 \mathrm{msec}$, which is characteristic for NMDAR EPSCs. When the NMDAR synaptic conductance is activated, $R_{\text {syn }}$ can be approximated by $R_{\text {syn }}(t)^{-1}=A\left\{-\exp \left(-\tau_{1} / t\right)+\right.$ $\left.\exp \left(-\tau_{2} / t\right)\right\}$, where $\tau_{1} \sim 8 \mathrm{msec}, \tau_{2} \sim 80 \mathrm{msec}$, and $A$ is an amplitude factor. In these conditions, a simple formula relates the time-dependent synaptic resistance $R_{\text {syn }}(t)$, and the conductance $Z_{\text {tot }}$ sampled by the amplifier:

$$
Z_{\text {tot }}(t)^{-1}=\left\{R_{\text {pip }}+\left(R_{\text {syn }}(t)^{-1}+Z_{\mathrm{e}}^{-1}\right)^{-1}\right\}^{-1},
$$

where $Z_{\mathrm{e}}=R_{\text {syn }} X_{\mathrm{C}} /\left(R_{\text {syn }}+X_{\mathrm{C}}\right)$ is the equivalent membrane impedance representing $R_{\mathrm{m}}$ and $C_{\mathrm{m}}$ combined. See Results and Figure 4 for additional details.

\section{Results}

\section{Independence of afferent pathways in stratum radiatum}

We made a 1.5- to 2-mm-long cut in stratum radiatum, parallel to stratum pyramidale, in rat hippocampal slices to separate two populations of axons (Fig. 1A). To test for presynaptic interactions between the pathways, we used a paired-pulse facilitation (PPF) protocol. When two stimuli were applied to one pathway with a $50 \mathrm{msec}$ interval, both AMPA receptor (AMPAR) and NMDAR EPSCs exhibited PPF $\left(\mathrm{EPSC}_{2} / \mathrm{EPSC}_{1}\right.$, mean \pm SEM:

$184 \pm 7$ and $145 \pm 7 \%, n=16$ and $n=18$, respectively) (Fig. $1 B, C$ ). In contrast, when the different pathways were alternately stimulated with the same interval, no cross-facilitation could be detected (101 \pm 2 and $93 \pm 4 \%$ for AMPAR- and NMDAR-mediated responses, respectively). The results imply that stimulation of one pathway did not recruit the fibers of the other pathway. Comparison of the mean PPF confidence limits for AMPAR responses indicates that, with a probability of $>0.98$, this cross-pathway contamination was $<5 \%$. [In the experiments in which two stimuli were applied to the same pathway, the facilitation of AMPARmediated EPSCs was 1.9-fold larger than that of NMDAR-mediated responses (see above; $p<0.001)$. Among possible mechanisms are that "NMDAR-only" synapses may have a different release probability (Poncer and Malinow, 2001) and that NMDA receptors occupied by glutamate released on the first stimulus are unavailable to mediate an incremental response to the second stimulus. We cannot exclude, however, imperfect recovery of the synaptic current arising from local shunting.] We observed a small but significant depression of the NMDAR responses when the two stimuli were applied to the different pathways (Fig. 1C) (cross-pathway stimulation; $93 \pm$ $4 \% ; p<0.05$ ). This depression was consistent with the hypothesis that high-affinity NMDARs (in contrast to low-affinity AMPARs) at one pathway bind glutamate released from the other pathway, thus resulting in partial occlusion of the response 50 msec after release. This temporal pooling is unlikely to apply to AMPARs because they have a faster off-rate (which is also evident from the AMPAR EPSC traces returning to the baseline long before the second stimulus) (Fig. $1 B$ ). An additional explanation for the small occlusion of the second NMDAR response is local shunting of synaptic currents (see below).

\section{Heterosynaptic activation of NMDARs detected with MK801}

Having established the independence of the two pathways converging on the same cell, we used the use-dependent NMDAR blocker MK801 (4 $\mu \mathrm{M})$ to look for cross talk among the NMDARs mediating EPSCs in the two pathways (Carter and Regehr, 2000). The blocker was bath applied after recording a sample of baseline $\operatorname{NMDAR}$ EPSCs $\left(V_{\mathrm{m}}=+40 \mathrm{mV}\right)$ elicited by stimulating the two pathways alternately. Single stimuli (25-55) 10-15 sec apart were then applied to one, conditioning pathway (CP) in the continued presence of MK801 until the EPSCs had decreased by $70-85 \%$ (on average, to $\sim 22 \%$ of baseline). Between stimuli, cells were held at $V_{\mathrm{m}}=-70 \mathrm{mV}$ to reduce spontaneous activation of NMDARs. The choice of CP and silent pathway (SP) was random. After 5 min washout of MK801, we resumed stimulation of the SP to determine the degree of reduction in NMDARmediated EPSCs caused by MK801 blockade. Only the first eight responses after washout were used for analysis, because MK801 washout was usually incomplete and responses often continued to decrease (see Materials and Methods). Their mean amplitude was reduced by $56 \pm 6 \%$ relative to baseline $(n=8 ; p<0.001)$ (Fig. 2A,C).

To test whether this reduction can be explained by the spontaneous activation of NMDARs while MK801 was present in the bath, we interleaved these experiments with others in which nei- 
ther pathway was stimulated in the presence of MK801. The protocol was otherwise identical. The average reduction of EPSCs in these "no-stimuli" control experiments was $28 \pm 6 \%(n=15 ; p<$ 0.001) (Fig. $2 B$ ), half of the $56 \%$ EPSC reduction in the $\mathrm{SP}$ after $\mathrm{CP}$ stimulation $(p<0.02)$. This implies that neurotransmitter molecules escaping from CP synapses activate NMDARs in the SP.

Because glycine is an obligatory coagonist of NMDARs, the NMDARmediated cross talk could occur, in principle, through the release and spillover of glycine rather than glutamate. To test for this possibility, we applied a saturating concentration of the glycine-binding site agonist D-serine $(100 \mu \mathrm{M})$. This had no effect on either the EPSC amplitude (98 \pm $7 \%$ of baseline; $n=10$ ) or its decrease in MK801 in no-stimuli control experiments $(69 \pm 5 \%$ of baseline compared with $72 \pm$ $6 \%$ of baseline in the previous experiments; $n=6$ and $n=15$, respectively; exposure to MK801 was similar in all experiments). These data indicated that the activity of glycine-binding sites had no effect on NMDAR activation in our experiments.

If the MK801 blockade of NMDARs in the SP was caused by spillover of glutamate from the CP, this blockade should be enhanced when glutamate release is enhanced or when glutamate uptake is inhibited (Asztely et al., 1997; Carter and Regehr, 2000; Diamond, 2001). We applied trains of five stimuli at $20 \mathrm{~Hz}$ instead of single stimuli to the CP while maintaining the same duration of exposure of NMDARs to MK801. In this protocol, the release probability increase factor, estimated in separate experiments as the average amplitude of five AMPAR-mediated responses (AMPAR responses were required to exclude temporal pooling of NMDAR EPSCs) relative to the single (first) response amplitude in the train, was $1.22 \pm$ $0.08(n=9 ; p<0.03)$ (Fig. $2 C$, inset). The EPSC amplitude in this pathway was reduced to $10-15 \%$ of baseline (on average, to $\sim 12 \%$ of baseline). Accordingly, the SP responses were reduced to $28 \pm 6 \%$ of baseline $(p<0.001 ; n=8)$, indicating an approximately twofold enhancement of the NMDAR blockade in the SP compared with that seen with single stimuli. The duration of the exposure to MK801 and the voltage steps applied to the postsynaptic cells were as for the previous experiments using single pulses, implying that the same no-stimuli control data apply.

Clearly, any synaptic cross talk should depend on the density of release sites or activated synapses, or both. Does the measure of spillover used here (reduction in the SP NMDAR responses postMK801) reflect this? Figure $2 D$ shows that the decrease postMK801 in the SP EPSCs (the measure of spillover) was indeed positively correlated $(r=0.60 ; p<0.03$ ) with the baseline EPSC amplitude in the $\mathrm{CP}$ (the measure of synaptic activation leading to spillover). This indicates that the extent of cross talk does increase, as expected, with the density of activated synapses. This prompts the question, however, of whether the densities of activated synapses in our experiments fall within the physiological range. Although it is impossible to reproduce the normal activity of the hippocampal circuitry in vitro, we took a simple approach by asking how the stimuli compared with the firing threshold of CA1 pyramidal cells (Arnth-Jensen et al., 2002). To estimate the action potential generation threshold, we recorded in cellattached mode (without glutamate receptor blockers in the bath) and gradually increased the extracellular stimulus strength until an action potential could be detected (Fig. $2 D$, inset). Once the threshold was established, the membrane was broken, and NMDAR EPSCs were then recorded in whole-cell mode, using the same stimulus strength (with AMPA-kainate receptors blocked as in the cross-talk experiments described above). The average amplitude of the NMDAR EPSCs that corresponded to the cell firing threshold was $257 \pm 82 \mathrm{pA}(n=9)$ (Fig. $2 D$, arrow). This was almost twice the average baseline amplitude of the CP NMDAR responses in the cross-talk experiments $(140 \pm 29 \mathrm{pA}$; 


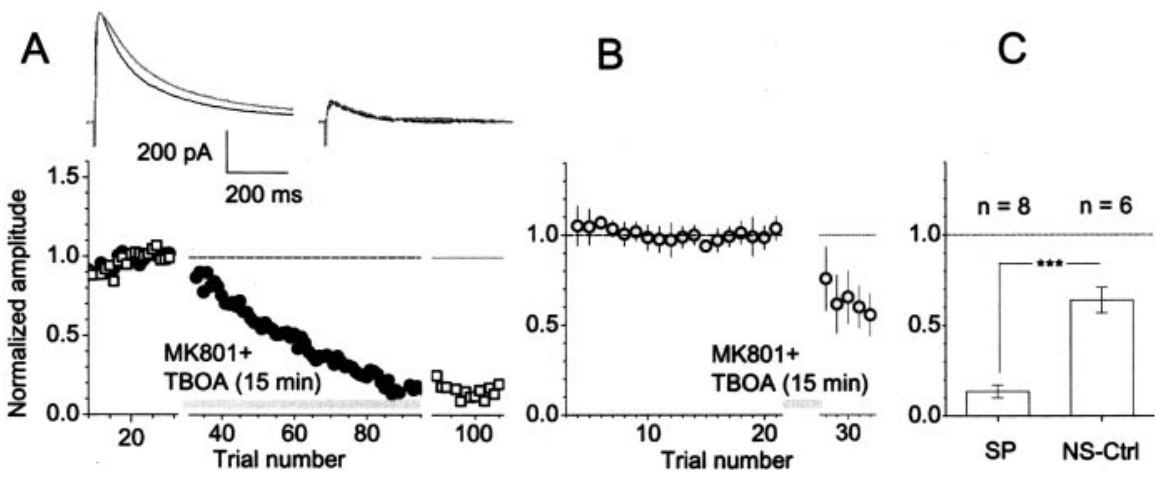

Figure 3. Blockade of glutamate uptake enhances synaptic cross talk. $A$, One cell example; filled circles and open squares, normalized amplitude of NMDAR EPSCs elicited by stimulation of CP and SP, respectively; gray segment, application of $4 \mu \mathrm{M}$ MK801 together with $50 \mu \mathrm{M}$ TB0A; traces, average EPSCs in CP (black) and SP (gray) before application (left panel; 20 last traces averaged) and after washout (right panel, first 8 averaged after washout). $B$, Normalized NMDAR EPSC amplitude in no-stimuli control experiments; summary data across cells $(n=6)$; error bars represent SEM. C, Statistical summary: average normalized NMDAR EPSC amplitude after application of $4 \mu \mathrm{m}$ MK801 together with $50 \mu \mathrm{m}$ TBOA in the SP and in no-stimuli control (NS-Ctrl, difference at $p<0.001)$.

$n=9$; single stimulus experiments only). Thus, assuming linear scaling, the number of synapses activated in the CP corresponded, on average, to 140 of $257=54 \%$ of the synaptic density required to fire a pyramidal cell. Although this protocol cannot fully reproduce the conditions of spike generation in vivo (not the least because the interneuron network may alter the excitability of the circuitry), the expected asynchrony of firing in vivo might require an even higher density of synapses activated within short time intervals for the postsynaptic cell to fire.

\section{Blocking glutamate uptake enhances cross talk}

We then tested the effect of the glutamate uptake blocker TBOA $(50 \mu \mathrm{M})$ on the extent of cross talk. To match the conditions of these experiments to those of the original experiments, we applied TBOA only during perfusion with MK801. NMDAR EPSCs in the SP were decreased to $14 \pm 4 \%$ of baseline, whereas the CP responses were decreased to $8 \pm 2 \%$ of baseline $(n=8)$ (Fig. 3). To test whether the more profound reduction was caused by the enhanced spontaneous MK801 blockade of NMDARs in the presence of TBOA rather than by enhanced spillover, we conducted the corresponding no-stimuli control experiments. After washout of TBOA plus MK801, EPSCs in these experiments were decreased to $64 \pm 7 \%$ of baseline (Fig. $3 C)(n=6$; same exposure to MK801 as in the preceding experiments). This was not significantly different from the effect of MK801 in the no-stimuli experiments without TBOA $(72 \pm 6 \%)$ (Fig. 2). We then further tested whether any long-term effects of TBOA alone (after washout) result in an increase in EPSCs. This could undermine the validity of the observed changes post-MK801. When TBOA alone was applied and, after $15 \mathrm{~min}$, washed out again, NMDARs EPSCs showed a modest reduction (to $79 \pm 8 \%$ of baseline; $n=6$ ) rather than an increase. This indicated that, if anything, the discrepancy between $\mathrm{CP}$ and no-stimuli control could have underestimated (by $\sim 20 \%$ ) the extent of synaptic cross talk in the presence of TBOA.

Thus, either increasing the amount of glutamate released or inhibiting glutamate uptake led to an enhanced blockade of the NMDA receptors mediating the SP EPSCs.

MK801 increases the diffusional distance from release sites to available NMDARs in the conditioning pathway

Because the concentration of glutamate after release decreases sharply outside the synaptic cleft, MK801 should block a decreas- ing proportion of NMDARs with increasing distance from the release site. When the synapse is activated repeatedly in the presence of MK801, the average diffusional distance from the release site to the remaining NMDARs, and hence the latency of the EPSCs, should therefore increase progressively. This increase is not expected, however, at synapses where NMDARs are activated by glutamate spillover from remote synapses (Fig. 4A).

Is it biophysically plausible to detect such an effect? To assess this, we used a detailed 3D model of a "typical" CA1 synapse (Fig. $4 B$ ). First, we simulated the extracellular glutamate concentration transient at several distances from the release site, inside and outside the cleft (Fig. 4C) (see Materials and Methods for details). This transient was then used to estimate the open probability of NMDARs at the corresponding loci. These simulations predict (Fig. 4D) that the NMDAR EPSC latency (70\% peak point) near the synaptic cleft edge in area CA1 should be $1.0-1.5 \mathrm{msec}$ greater than that near the synaptic cleft center (the release site). At a distance of 200$250 \mathrm{~nm}$, which is compatible with the half-distance between nearest neighbors in the CA1 neuropil (Rusakov and Kullmann, 1998), the latency increase should be $2.0-2.5 \mathrm{msec}$. These time intervals are well within the resolution of electrophysiological recordings. Indeed, an increase in the NMDAR EPSC rise time has been reported to occur after application of MK801 (ArnthJensen et al., 2002).

The 70\% peak latency of CP EPSCs should thus increase after application and washout of MK801. In keeping with the prediction of an increased diffusion distance, we observed a latency increase of $3.18 \pm 0.49 \mathrm{msec}$ in the $\mathrm{CP}(p<0.001 ; n=10)$ (Fig. $4 E$, top bars). In these experiments, however, the SP EPSCs also showed a latency increase $(1.13 \pm 0.39 \mathrm{msec})$ (Fig. $4 E$, top bars), albeit three times smaller than the increase in the $\mathrm{CP}(p<0.001$; $n=10)$. This increase could not be readily explained by voltageclamp escape because smaller responses are expected to be better clamped and therefore exhibit faster, rather than slower, rise times. Moreover, because the MK801 blockade should not affect the spatial pattern of release sites, dendritic filtering is also unlikely to affect the EPSC shape.

Another phenomenon that can affect the measurement of the early part of the NMDAR EPSC is incomplete washout of MK801. The EPSC decay time constant was markedly shortened by MK801, as expected from a shortening of the average NMDAR open time, and this effect was only partially reversed after washout, implying that MK801 was still present (Fig. $4 E$, traces). This is predicted to have the indirect effect of shortening the rise time of the synaptic conductance, because it should peak earlier than in the absence of MK801 (Jahr, 1992; Rosenmund et al., 1993); however, this effect is opposite to the observed change, which was an increase in the $70 \%$ peak latency $(\sim 3 \mathrm{msec}$ in the $\mathrm{CP}$ and $\sim 1$ msec in the SP). Moreover, although the expected small shortening in the rise time of the conductance waveform could lead to an underestimate of the EPSC latency increase, it should affect both pathways equally (because it is a function not of cumulative blockade but of the acute effect of MK801 persisting at the end of the experiment). Thus, this phenomenon should not introduce any bias to the comparison between pathways. 
An alternative phenomenon must therefore underlie the increase in latency in the SP. A possible explanation for the nonspecific latency increase is that the peak of the NMDAR EPSC was distorted by local shunting of the synaptic current through open NMDA receptors.

To address this further, we performed an equivalent circuit analysis of the voltage-clamp configuration representative of our experiments (Fig. $4 F$ ) (see Materials and Methods for derivations and parameter values; for the purposes of shunt analysis, ideal voltage clamp and no dendritic filtering were assumed). The analysis predicts that, given a characteristic synaptic conductance of $2-3 \mathrm{nS}$ (20-30 activated NMDARs at each synapse) and given the typical conditions of our experiments (50-100 activated synapses or 100-300 nS total conductance), a threefold decrease in NMDAR EPSCs corresponds to a $\sim 1 \mathrm{msec}$ increase in its $70 \%$ peak latency (Fig. 4G). (In this range of amplitudes, the NMDAR EPSC amplitude error attributable to sublinear summation is $<10-15 \%$.) This change in latency is in excellent agreement with the experimental data for the nonspecific increase in latency in the SP.

The question arises, however, whether this shunting could then account for the differential latency increases in the two pathways simply because the EPSC amplitude decreased more in the CP than in the SP. To test for this possibility, we applied the NMDAR antagonist $R(-)$-3-(2-carboxypiperazin-4-yl)-propyl-1-phosphonic acid (CPP) in a nonsaturating concentration $(2.5-5 \mu \mathrm{M})$ that reduced the baseline responses to a similar extent as MK801 in the CP (17 $\pm 4 \%$ ) (Fig. $4 E$, bottom graph). The latency increase in these responses was half that seen in the post-MK801 CP responses of similar size $(1.48 \pm 0.48 \mathrm{msec}$, $n=12$; compared with $3.18 \pm 0.49 \mathrm{msec}$, $n=10 ; p<0.04)$. Thus, although local synaptic current shunting may explain part or all of the nonspecific latency increase seen in the SP, the additional latency increase observed in the CP can be attributed to an increased diffusional distance from the release site to the remaining unblocked receptors.

To further verify that the difference between the actions of MK801 and CPP was not caused by differential penetration of the drugs into the synaptic cleft, we also measured the latency of EPSCs after application of MK801 together with TBOA (see above). TBOA should increase the proportion of remote NMDARs activated by glutamate (Lozovaya et al., 1999; Diamond, 2001) and therefore subsequently blocked by MK801. Thus, when TBOA plus MK801 are washed out, a relatively higher proportion of intrasynaptic versus extrasynaptic receptors

B
C

E
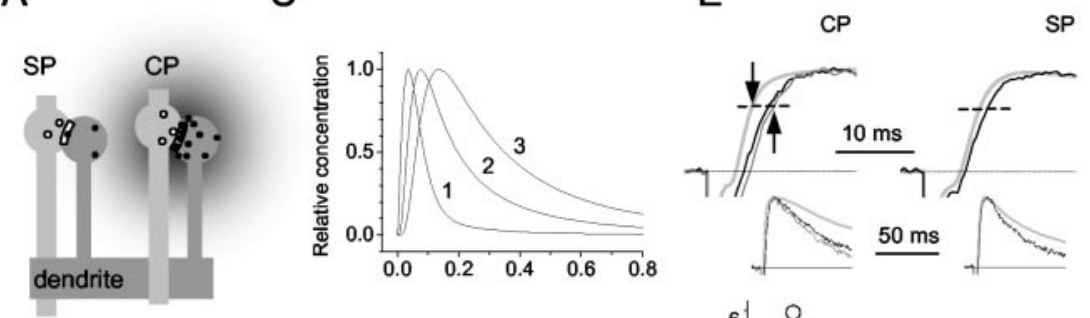

D
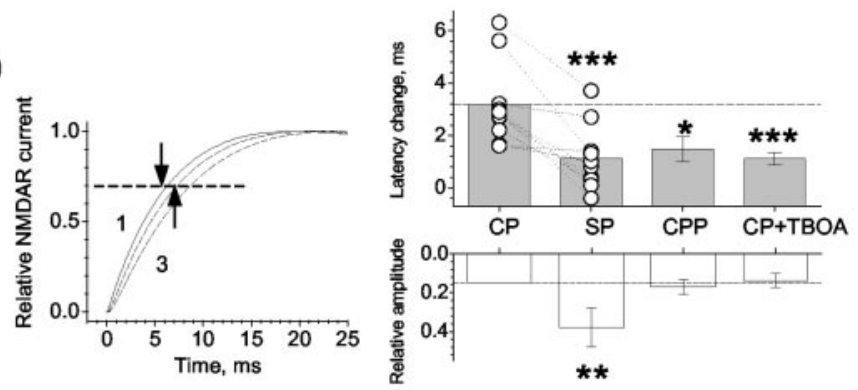

G

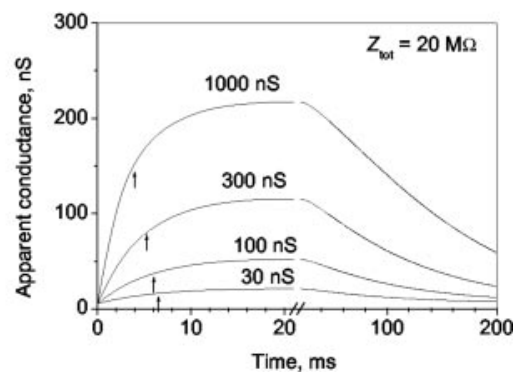

Figure 4. Blockade by MK801 increases the average distance from release site to NMDARs at the directly activated CP synapses but not spillover activated SP synapses. A, Diagram: synaptic releases (gray shadow) at the (P axo-spine synapses (en passant boutons and dendritic spines are depicted) activate a higher proportion of NMDARs (shown as black dots) near the release site, whereas spillover-mediated activation of NMDARs at the neighboring SP is homogeneous. B, A simplified diagram depicting the 3D model of synaptic environment (Lehre and Rusakov, 2002) (see Materials and Methods). The synaptic apposition zone is enclosed between two hemispheric obstacles to diffusion, which are separated from the surrounding porous neuropil by an extracellular gap. Glial glutamate transporters are distributed unevenly (shown as a postsynaptic shadow) to match experimental data (Lehre and Danbolt, 1998; Lehre and Rusakov, 2002). C, Simulated time course of glutamate diffusion transient (amplitude is normalized) near the release site (1, synaptic cleft center), cleft edge (2), and at $250 \mathrm{~nm}$ from the release site (3). D, NMDAR activation time course (initial fragment; amplitude is normalized) simulated from the corresponding glutamate diffusion transients ( $C$ and the detailed kinetics of NMDAR activation (Lester and Jahr, 1992). Dotted line and vertical arrows depict the $70 \%$ peak latency for the NMDAR activation near the release site and at the cleft edge (notations 1 and 3 correspond to those in C; line 2 is between 1 and 3). E, Traces: the average NMDAR response (amplitude is normalized) evoked by stimulation of the CP and SP (as indicated) before, in the presence of (CP only), and after washout of MK801 (thick light gray, thin dark gray, and black line, respectively); insets, same traces at a longer time scale; average of 20 traces. Top bar graphs indicate average changes in the $70 \%$ peak latency of NMDAR EPSCs after application and washout of MK801 in the (P and SP (as indicated; $n=10$ ), in 2.5-5 $\mu \mathrm{M}$ (PP (CPP; single pathway experiments; $n=12)$, and in the (P in the presence of TBOA (CP + TBOA; $n=6)$, as indicated. Bottom bar graphs indicate the corresponding EPSC amplitude (normalized relative to baseline in each experiment). $F$, An equivalent (approximate) circuit of voltage-clamp experiments (see Materials and Methods). Inset, because the cell membrane reactance $X_{C}$ is not apparent in experimental measurements, it is convenient to combine $R_{\mathrm{m}}$ and $C_{\mathrm{m}}$ as the equivalent impedance $Z_{\mathrm{e}}=$ $R_{\text {syn }} X_{C} /\left(R_{\text {syn }}+X_{C}\right) \cdot G$, The time course of the total conductance $Z_{\text {tot }}$ recorded by the amplifier when $R^{-1}$ syn $(t)$ follows the kinetics of NMDAR EPSC, $A\left\{-\exp \left(-\tau_{1} / t\right)+\exp \left(-\tau_{2} / t\right)\right\}$ (see Materials and Methods), for different values of the amplitude factor $A$, as indicated. Arrows indicate the $70 \%$ peak time point. The abscissa is divided into two scales.

should be available to synaptically released glutamate. This implies a smaller increase in EPSC latency compared with the case of MK801 application without TBOA. In accordance with this prediction, the latency increase of the CP EPSCs was substantially smaller than that observed without application of TBOA (Fig. $4 E$, top bar graph) $(1.13 \pm 0.28 \mathrm{msec} ; n=7 ; p<0.001$; latency increase in SP: $1.72 \pm 0.59 \mathrm{msec}$; data not shown), whereas the EPSC amplitude was reduced to the same extent (Fig. $4 E$, bottom bar graph) (14 $\pm 4 \%$ of baseline). These data therefore support the hypothesis that the MK801 blockade increases the average 
A $\quad$ B $\quad$ D
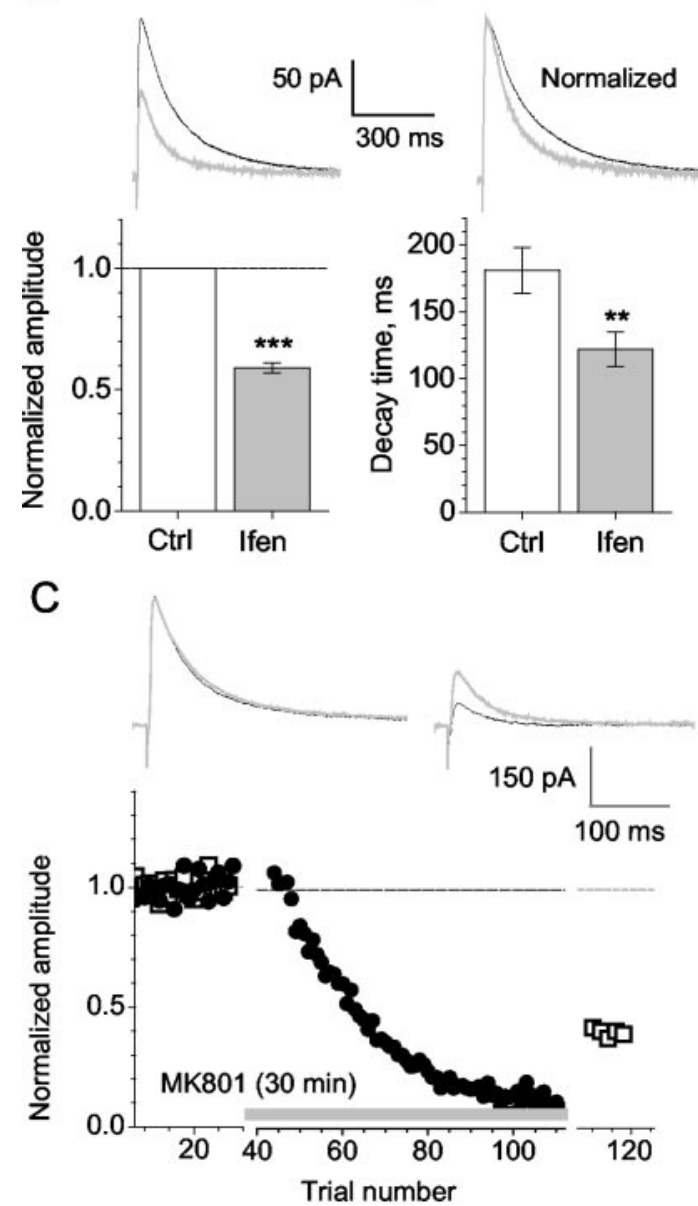

No-stimulus control

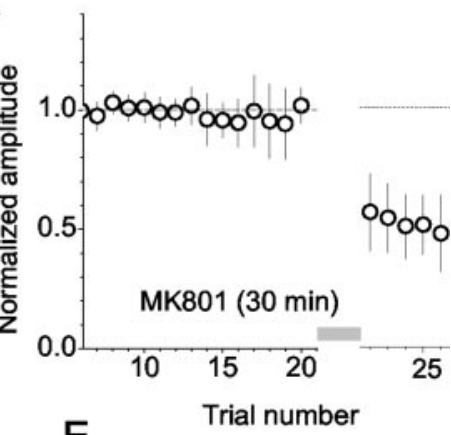

$\mathrm{E}$

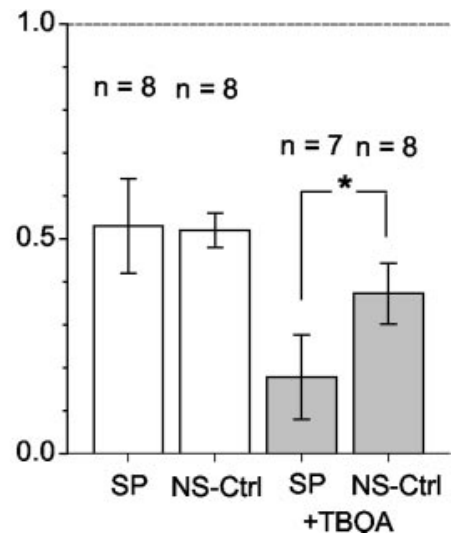

Figure 5. Blocking NR2B-containing NMDARs diminishes intersynaptic cross talk, whereas TBOA rescues it. $A, B$, Ifenprodil reduces the amplitude $(A)$ and shortens the decay $(B)$ of evoked NMDAR EPSCs. Traces show one cell example (average of 20 sweeps) in control (black) and ifenprodil (gray); traces in $B$ are normalized to the same peak value; bar plots indicate statistical summary for the measurements of amplitude $(A)$ and decay $(B)(n=16)$; see Results for details. C, Two-pathway experiment in the continued presence of ifenprodil: one cell example; filled circles and open squares, normalized amplitude of NMDAR EPSCS elicited by stimulation of CP and SP, respectively; gray segment, application of $4 \mu \mathrm{m} \mathrm{MK801;} \mathrm{traces,} \mathrm{average} \mathrm{EPSCs} \mathrm{in} \mathrm{CP} \mathrm{(black)}$ and SP (gray) before application (left panel; 20 last traces averaged) and after washout (right panel; first 8 averaged after washout). D, Normalized NMDAR EPSC amplitude in no-stimuli control experiments; summary data across cells $(n=8)$; error bars represent SEM. E, Statistical summary. White bars: average normalized NMDA EPSC amplitude after application of $4 \mu \mathrm{m}$ MK801, in the continued presence of ifenprodil, in the SP $(n=8)$, and in no-stimuli control (NS-Ctrl); gray bars: similar experiments but with MK801 applied together with TBOA $(p<0.03)$.

distance between release sites and NMDARs at the directly activated (CP) but not spillover-activated (SP) synapses. On the basis of the predictions of modeling (Fig. $4 D$ ), the observed difference in NMDAR EPSC latency of 1.5-2 msec that cannot be accounted for by local shunting should correspond to a difference of $120-$ $200 \mathrm{~nm}$ in the typical diffusion distance.

\section{NR2A- and NR2B-containing NMDARs in intersynaptic signaling in CA1}

In the cortex, functional NMDARs must contain either a NR2A or a NR2B receptor subunit (Kutsuwada et al., 1992). The expression of NR2A-containing receptors, which are thought to be almost exclusively intrasynaptic, increases sharply with synaptic maturation (Tovar and Westbrook, 1999), with the NR2A/NR2B ratio reaching its maximum at postnatal day 28 (Kew et al., 1998). Several studies have argued that extrasynaptic receptors tend to contain NR2B subunits, whereas synaptic receptors can contain either subtype (Stocca and Vicini, 1998; Momiyama, 2000;
Steigerwald et al., 2000; Dalby and Mody, 2003; de Armentia and Sah, 2003). Although NR2B receptors have a two- to threefold higher sensitivity for glutamate compared with NR2A receptors (Kutsuwada et al., 1992), the progress of MK801 blockade in the presence of glutamate is indistinguishable between NR2Acontaining, NR2B-containing, and the "endogenous mixture" of NMDARs expressed in cultured neurons (Prybylowski et al., 2002). In the conditions of the present MK801 experiments, therefore, the selective NR2B blocker ifenprodil (Williams et al., 1993) is a useful tool for dissecting the relative contribution of these receptor subtypes to intersynaptic cross talk.

Application of ifenprodil $(10 \mu \mathrm{M})$ decreased the NMDAR EPSC amplitude to $58 \pm 5 \%$ of baseline $(p<0.001 ; n=16)$ (Fig. 5A). This decrease was in agreement with the effect of the selective NR2B antagonist Ro 25-6981 on NMDAR EPSCs in CA1 pyramidal cells reported recently in mice (Kawakami et al., 2003): decrease to 64 and $63 \%$ of baseline, respectively, in the left and right hemisphere. The decrease in EPSC amplitude produced by ifenprodil application was accompanied by a reduction in the monoexponential decay time constant from $181 \pm 17$ to $122 \pm 13 \mathrm{msec}$ $(p<0.002 ; n=16)$ (Fig. $5 B)$. This is consistent with the difference between decay time constants of NMDAR-mediated currents in NR2B- and NR2A-transfected cerebellar granule cells: $225 \mathrm{msec}$ and 116 msec, respectively (Prybylowski et al., 2002).

We repeated the two-pathway crosstalk experiment in the continued presence of ifenprodil, with the same MK801 exposure protocol as in previous experiments. The progress of NMDAR EPSC blockade in the CP appeared slower compared with the experiments without ifenprodil (reduction to $\sim 40 \%, n=6$, compared with $\sim 22 \%$ of baseline, respectively). This was consistent with the substantially lower activation sensitivity of the remaining NR2A-containing NMDARs (Kutsuwada et al., 1992); however, we cannot fully exclude the possibility that NR2B-containing NMDARs are more sensitive to MK801. The similarity in MK801 sensitivity between the two isoforms reported in culture (Prybylowski et al., 2002) might not fully capture the behavior of the receptors in hippocampal slices. An additional contributing factor could be a direct consequence of synaptic cross talk. If the higher-affinity NR2B receptors sense glutamate released from more remote sites, these receptors will be activated in a higher number of trials (because the release probability at CA1 synapses is substantially lower than unity) compared with local NR2A receptors.

This gives rise to a paradox. Although NR2A-containing NMDARs show a lower cumulative rate of blockade by MK801 than do NR2B-containing receptors (Fig. 5), the diffusional dis- 
tance increases in MK801 (Fig. 4), implying a higher occupancy of intrasynaptic versus extrasynaptic receptors. A likely resolution of this paradox is that a significant proportion of NR2Bcontaining receptors occur close to synapses. Such receptors should be blocked by MK801 more rapidly than either local NR2A-containing or remote NR2B-containing receptors because they are exposed to both local (high occupancy, low frequency) and spillover-mediated (low occupancy, high frequency) glutamate release. The rapid blockade of these receptors by MK801 therefore would increase the relative contribution of remotely activated NR2B NMDARs to the synaptic signal. The latter is consistent with the increased 70\% peak latency of EPSCs (Fig. 4).

To allow the CP EPSCs to decrease to a level comparable with that seen in experiments without ifenprodil (15-20\%), we increased the exposure to MK801 from 15 to $30 \mathrm{~min}$. With the longer exposure to MK801, the residual EPSC amplitude in the SP was $53 \pm 11 \%$ of baseline $(n=8)$ (Fig. 5C,E). Surprisingly, the NMDAR EPSCs underwent a similar average reduction in the corresponding no-stimuli experiments ( $52 \pm 4 \%$ of baseline; $n=8$; same exposure to MK801) (Fig. 5D,E). This implies that intersynaptic cross talk mediated by NMDARs was undetectable in the presence of $10 \mu \mathrm{M}$ ifenprodil.

Because $10 \mu \mathrm{M}$ ifenprodil potentially blocks a small fraction of NR2A receptors, we repeated these experiments in the presence of $3 \mu \mathrm{M}$ ifenprodil. This data set again yielded no evidence for cross talk. NMDAR EPSCs in the SP and no-stimulus control were reduced to $38 \pm 6 \%$ of baseline (post-MK801; $n=3$ ) and $36 \pm 3 \%$ of baseline $(n=6$; data not shown), respectively. The greater reduction compared with that in $10 \mu \mathrm{M}$ ifenprodil may reflect more rapid trial-to-trial progress of MK801 blockade under conditions in which fewer NR2B receptors are blocked.

In the presence of ifenprodil, only NR2A-containing NMDARs remain available to detect released glutamate. Because these receptors, unlike NR2B-containing NMDARs, appear to be restricted to synapses, one might also expect that there would be no specific latency increase after MK801 blockade in these conditions. The $70 \%$ peak latency of the post-MK801 EPSCs in the $\mathrm{CP}$ was increased only by $0.88 \pm 0.30 \mathrm{msec}$ relative to baseline $(n=6)$. In accordance with our predictions, this increase was actually smaller than the nonspecific increase of $1.13 \pm 0.39 \mathrm{msec}$ in similar experiments with CPP (although this difference fell short of statistical significance). Importantly, the latency increase was three times smaller than that without ifenprodil (3.18 \pm 0.49 ; $p<0.005)$.

Because blocking glutamate uptake with TBOA substantially increased the extent of synaptic cross talk (Fig. 3), we tested whether this manipulation could rescue, at least partly, the cross talk abolished by ifenprodil. When TBOA was applied together with ifenprodil, the discrepancy between the average EPSC amplitude at the SP and at the no-stimuli control was indeed partly restored $(18 \pm 10$ and $37 \pm 7 \%, n=7$ and $n=8$, respectively; $p<$ 0.03; the CP EPSC amplitude was $15 \pm 10 \%$ ) (Fig. 5E).

\section{Discussion}

\section{The extent of spillover in CA1}

The main results of this study are, first, that a proportion of NMDARs in CA1 pyramidal cells recorded at a nearphysiological temperature mediate excitatory signaling from more than one synapse and, second, that such receptors mainly, if not exclusively, contain the NR2B subunit.

These results allow a quantitative estimate for the extent of spillover. In the no-stimuli experiments the NMDAR EPSCs were reduced to $71 \%$ of the baseline, whereas this reduction was to $44 \%$ of baseline in the SP (Fig. 2C). Spillover can therefore ac- count for an MK801-dependent blockade of $100 \cdot(1-44 / 71)$ $38 \%$ of the SP NMDARs. This reduction occurred after $n=$ 25-55 stimuli to the CP. Because low-frequency stimulation is likely to involve principally synapses with a high release probability (Rosenmund et al., 1993), the proportion $p$ of NMDARs blocked by MK801 (in either pathway) per stimulus should not depend on $n$. Thus, the baseline-normalized amplitude of NMDAR EPSCs after the first stimulus will be $A(1)=(1-p)$ and the amplitude after the $n$th stimulus will be $A(n)=(1-p)^{n}$. Allowing indexes $c$ and $s$ for the CP and SP, respectively, one then obtains:

$$
\frac{p_{\mathrm{c}}}{p_{\mathrm{s}}}=\frac{1-A_{\mathrm{c}}^{1 / \mathrm{n}}(n)}{1-A_{\mathrm{s}}^{1 / \mathrm{n}}(n)} .
$$

Given $A_{\mathrm{c}}=0.3(22$ over $71 \%)$ and $A_{\mathrm{s}}=0.62(44$ over $71 \%)$, the formula yields a ratio close to 3 . In other words, there is an $\sim 30 \%$ chance that glutamate released from one pathway will successfully activate NMDARs in the other pathway. When groups of five stimuli were applied in our experiments, the value of $p_{c} / p_{s}$ became close to $2.0\left(A_{\mathrm{c}}=12 / 71=0.17 ; A_{\mathrm{s}}=24 / 71=0.39\right.$; total stimulus number $n \sim 120$ ), indicating an increased probability (50\% chance) of intersynaptic cross talk in CA1. In fact, because low-probability synapses may be recruited in this experiment, $50 \%$ is likely to be an underestimate. In TBOA (Fig. 3), cross talk was even more probable: $p_{\mathrm{c}} / p_{\mathrm{s}}$ was close to $1.35\left(A_{\mathrm{c}}=8 / 64=\right.$ $0.125 ; A_{\mathrm{s}}=14 / 64=0.219$; stimulus number $n \sim 40$ ).

\section{Sparseness of activated synapses and glutamate spillover}

Our results therefore predict that, on average, $25-35 \%$ of NMDARs in CA1 pyramidal cells sense glutamate released at more than one synapse, with the stimulus intensities and frequencies used here. Is this estimate biophysically plausible? To address this, it is necessary to estimate the density of activated synapses. Before applying CNQX to block AMPA receptors, we observed evoked EPSCs in the range of $A_{\text {evok }}=0.5-1 \mathrm{nA}$ and spontaneous EPSCs in the range of $A_{\text {spon }}=10-15 \mathrm{pA}$. Given that blocking action potentials with TTX has little effect on the amplitude of spontaneous EPSCs in CA1 pyramidal neurons (Manabe et al., 1992), this implies that a single stimulus activates $A_{\text {evok }} / A_{\text {spon }}=50-100$ synapses per cell. In area CA1, apical dendrites of pyramidal cells receive 5,000-10,000 synaptic inputs (Trommald et al., 1995), implying that up to $2 \%$ of excitatory synapses were activated. Because the volume density of all excitatory synapses in CA1 is $\sim 2 \mu \mathrm{m}^{-3}$ (Rusakov and Kullmann, 1998), the density of activated synapses will be $N_{\mathrm{V}} \sim 0.04 \mu \mathrm{m}^{-3}$. This corresponds to a nearest-neighbor distance among activated synapses of $0.554 N_{\mathrm{V}}{ }^{-1 / 3}=1.62 \mu \mathrm{m}$ (Rusakov and Kullmann, 1998).

Is this synaptic density consistent with the $25-35 \%$ chance of NMDARs being "shared" among synapses? To address this, we performed simulation experiments that would mimic the experimental protocol. We generated a $3 \mathrm{D}$ scatter of synapses in a 5 - $\mu \mathrm{m}$-wide cube of neuropil (Fig. $6 \mathrm{~A}$, schematic). The size, spatial density, and typical geometry of synaptic environment matched the experimental estimates obtained using electron microscopy (Rusakov and Kullmann, 1998; Lehre and Rusakov, 2002). A subset of activated synapses was sampled randomly (Fig. $6 \mathrm{~A}$, red and yellow spheres), and 5000 glutamate molecules (see Materials and Methods) were released synchronously at each of the selected synapses. The resulting spatial profile of glutamate at a given time point could then be visualized using a "probing plane" that crossed the cube (Fig. 6A,B). Conversely, the gluta- 
A

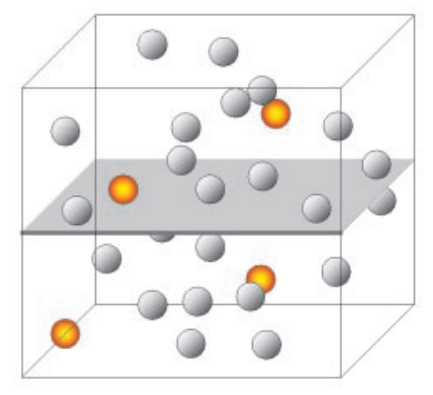

B

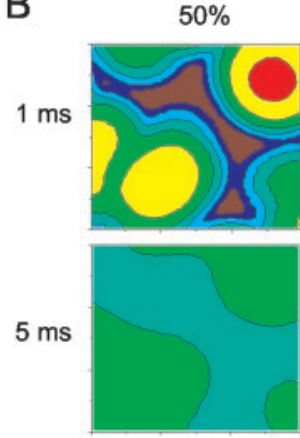

D

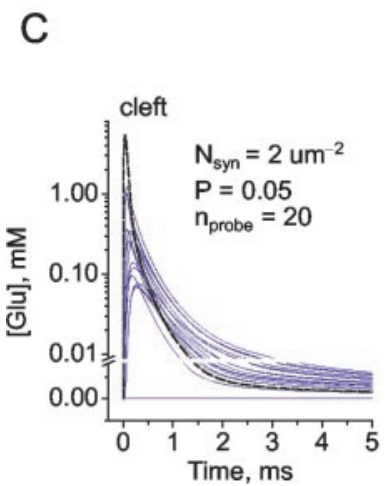

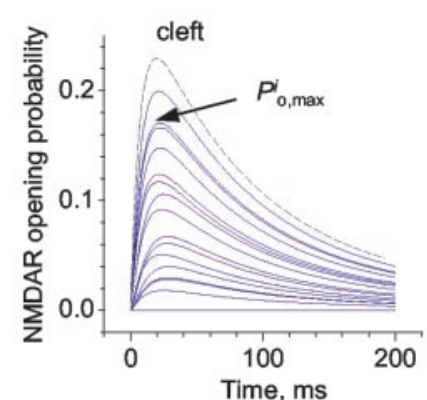

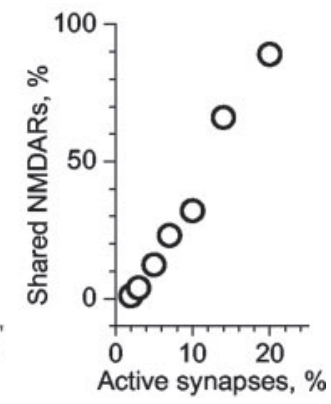

Figure 6. Simulated transients of glutamate in the 3D scatter of synaptic release sites. $A$, Diagram depicting simulation experiments (not to scale): 250 synapses were scattered as a hard-core Poisson process (spheres) within a $5 \mu \mathrm{m}$ wide cube; the chosen proportion of active synapses was sampled randomly (red and yellow spheres); after synchronous releases, each active synapse generated a 3D diffusion profile, in accordance with the model illustrated in Figure $2 B-D$ (Lehre and Rusakov, 2002). $B$, The resulting spatial profile of glutamate in the probing plane $(A) 1$ and $5 \mathrm{msec}$ after a synchronous release of glutamate from a 50 and $10 \%$ subset of synapses, as indicated. Note the logarithmic color scale of concentrations. C, Time course traces of extracellular glutamate simulated at an arbitrary site in the neuropil during 20 trials when a $5 \%$ subset of active synapses was resampled randomly at each trial; each line represents spatial superimposition of glutamate transients originating at individual synapses. Dotted line indicates, for comparison, calculated glutamate time course within the synaptic cleft; 3 of 20 traces indicated negligible diffusion transients (shown as zero lines). The ordinate axis is broken into two scales. D, The NMDAR opening kinetics (Lester and Jahr, 1992), which corresponds to glutamate transients shown in C; dotted line shows the kinetics inside the synaptic cleft. The arrow shows the peak opening probability of NMDARs $\left(P_{0, \max }^{i}\right)$ at the ith trial. $E$, The average peak opening probability, $\left\langle P^{i}{ }_{0, \max } \cdot P^{j}{ }_{0, \max }\right\rangle$, of NMDARs that would be activated by (shared between) both of any two randomly sampled synaptic subsetsindependent pathways, plotted against the proportion of activated synapses.

mate concentration transient and the corresponding NMDAR opening time course [estimated from the kinetic scheme of Lester and Jahr (1992)] could be simulated at a given location within the cube. To mimic the two-pathway experiments, we collected such recordings at an arbitrary point, whereas the subset of active synapses was randomly resampled at each trial (see Materials and Methods). Figure 6, $C$ and $D$, depicts a sample $(n=20)$ of glutamate transients and the corresponding NMDAR activation curves, respectively, generated by the $5 \%$ subsets of synapses. In such simulations, the $i$ th trial would correspond to an NMDAR maximum opening probability $P_{\text {o,max }}^{i}(i=1, \ldots, 20)$ in response to glutamate release from the $i$ th subset of synapses. The probability that the same NMDARs will be activated by both of any two independent synaptic subsets could then be calculated as the product $P_{i j}=P_{\text {o,max }}^{i} P^{j}{ }_{\text {o,max }}(i \neq j)$, with indices $i$ and $j$ representing two independent pathways. Figure $6 E$ shows the results of such simulations depicting the expected average $\left\langle P_{i j}\right\rangle$ plotted against the proportion of activated synapses.

These data suggest that $\sim 30 \%$ of NMDARs will be shared among independent synaptic subsets when 7-8\% of all local synapses are activated. This compares with the estimate, obtained from the electrophysiological data, that only $2 \%$ of synapses were active (see above). The latter estimate, however, reflects the average value throughout the neuropil, whereas in the acute slice functional synapses are likely to be distributed unevenly (for instance because some dendrites were cut during slicing), thus giving higher local densities. The predictions of the modeling are thus in reasonable agreement with the electrophysiology.

\section{The role of NR2B- and NR2A- containing receptors in spillover}

Our data predict that blocking the NR2Bcontaining NMDARs with ifenprodil effectively abolishes activation of NMDARs by escaping glutamate. The lower sensitivity of the NR2A-containing NMDARs to glutamate (Kutsuwada et al., 1992) provides one possible explanation; however, this could still give rise to a paradox. Because there are many inactive synapses ( $\sim 95 \%$ of the total) scattered between active ones, some NR2A-containing receptors could be in relatively close proximity to release sites. One major distinction between the two receptor subunits, however, is that the NR2A-containing NMDARs are thought to occur close to the release site, perhaps exclusively within the synaptic cleft (Tovar and Westbrook, 1999; Momiyama, 2000; Steigerwald et al., 2000; Dalby and Mody, 2003; de Armentia and Sah, 2003). It is conceivable therefore that these receptors are surrounded by highaffinity receptors or transporters, or both, expressed in the adjacent neuronal or glial membranes. [The function of postsynaptic transporters was probably compromised by holding the cell at a positive voltage, arguing instead for a role of presynaptic transporters (Danbolt, 2001; Mathews and Diamond, 2003).] In this case, the intracleft NR2A-containing receptors would be protected from lowconcentration waves of glutamate spilling in (Diamond, 2001). In agreement with this hypothesis, blocking glutamate uptake with TBOA partly rescued the discrepancy between the $\mathrm{CP}$ and no-stimuli control (Fig. 5E), thus removing, at least in part, the barrier for intersynaptic cross talk mediated by NR2A-containing NMDARs.

Finally, the finding that synaptic cross talk depends on NR2B receptors argues against major contribution of glutamate released from glia (Araque et al., 1999) to the observed spillover phenomena. A substantial proportion of synaptic clefts (and therefore NR2A-containing NMDARs) in CA1 are in the immediate proximity of glial protrusions (Ventura and Harris, 1999).

\section{Functional implications of intersynaptic cross talk}

The conclusion that receptors activated by transmitter release at one synapse can also be activated by another synapse implies a significant loss of information capacity in the network; however, this issue is not straightforward. First, low-affinity AMPARs can still mediate fast, point-to-point synaptic transmission without degradation of spatiotemporal "bandwidth." Second, even 
among high-affinity NMDARs, the present study implies that it is NR2B-containing receptors that are shared among multiple afferent inputs. This is in line with the notion that the extrasynaptic NMDARs, which are supposed to contain the NR2B subunit, are distributed over a 100-fold larger area (although at a much lower concentration) (Takumi et al., 1999; Racca et al., 2000) of cell membranes compared with the synaptic cleft (Rusakov et al., 1998).

Interestingly, NR2A- and NR2B-containing NMDARs have been linked to different intracellular cascades (Steigerwald et al., 2000; Zheng et al., 2001; Krapivinsky et al., 2003), also leading to preferential triggering of long-term potentiation and long-term depression, respectively ( $\mathrm{Lu}$ et al., 2001; Hardingham et al., 2002). Thus, the results of the present study suggest a possible novel role for NR2B receptors. Because they detect glutamate released from multiple sources, they may exist primarily to monitor the overall level of activity in the network (Dalby and Mody, 2003) and thus to regulate the density and strength of glutamatergic synapses (Turrigiano, 1999). Indeed, the present data suggest a simple scenario in which a proportion of NR2B-containing NMDARs are activated by glutamate spilled over from synapses on neighboring cells. In contrast, NR2A-containing NMDARs do not appear to share such spillover signals, thus suggesting a distinction between "global" and "local" signaling sensed by distinct subpopulations of NMDARs.

\section{References}

Araque A, Parpura V, Sanzgiri RP, Haydon PG (1999) Tripartite synapses: glia, the unacknowledged partner. Trends Neurosci 22:208-215.

Arnth-Jensen N, Jabaudon D, Scanziani M (2002) Cooperation between independent hippocampal synapses is controlled by glutamate uptake. Nat Neurosci 5:325-331.

Asztely F, Erdemli G, Kullmann DM (1997) Extrasynaptic glutamate spillover in the hippocampus: dependence on temperature and the role of active glutamate uptake. Neuron 18:281-293.

Barbour B (2001) An evaluation of synapse independence. J Neurosci 21:7969-7984.

Bergles DE, Jahr CE (1998) Glial contribution to glutamate uptake at Schaffer collateral-commissural synapses in the hippocampus. J Neurosci 18:7709-7716.

Carter AG, Regehr WG (2000) Prolonged synaptic currents and glutamate spillover at the parallel fiber to stellate cell synapse. J Neurosci 20:4423-4434.

Clark BA, Cull-Candy SG (2002) Activity-dependent recruitment of extrasynaptic NMDA receptor activation at an AMPA receptor-only synapse. J Neurosci 22:4428-4436.

Cossart R, Tyzio R, Dinocourt C, Esclapez M, Hirsch JC, Ben-Ari Y, Bernard C (2001) Presynaptic kainate receptors that enhance the release of GABA on CA1 hippocampal interneurons. Neuron 29:497-508.

Dalby NO, Mody I (2003) Activation of NMDA receptors in rat dentate gyrus granule cells by spontaneous and evoked transmitter release. J Neurophysiol 90:786-797.

Danbolt NC (2001) Glutamate uptake. Prog Neurobiol 65:1-105.

de Armentia ML, Sah P (2003) Development and subunit composition of synaptic NMDA receptors in the amygdala: NR2B synapses in the adult central amygdala. J Neurosci 23:6876-6883.

Diamond JS (2001) Neuronal glutamate transporters limit activation of NMDA receptors by neurotransmitter spillover on CA1 pyramidal cells. J Neurosci 21:8328-8338.

Franks KM, Bartol TM, Sejnowski TJ (2002) A Monte Carlo model reveals independent signaling at central glutamatergic synapses. Biophys J 83:2333-2348.

Hardingham GE, Fukunaga Y, Bading H (2002) Extrasynaptic NMDARs oppose synaptic NMDARs by triggering CREB shut-off and cell death pathways. Nat Neurosci 5:405-414.

Harris KM, Sultan P (1995) Variation in the number, location and size of synaptic vesicles provides an anatomical basis For the nonuniform probability of release at hippocampal CA1 synapses. Neuropharmacology 34:1387-1395.
Jahr CE (1992) High probability opening of NMDA receptor channels by L-glutamate. Science 255:470-472.

Kawakami R, Shinohara Y, Kato Y, Sugiyama H, Shigemoto R, Ito I (2003) Asymmetrical allocation of NMDA receptor $\epsilon 2$ subunits in hippocampal circuitry. Science 300:990-994.

Kew JNC, Richards JG, Mutel V, Kemp JA (1998) Developmental changes in NMDA receptor glycine affinity and ifenprodil sensitivity reveal three distinct populations of NMDA receptors in individual rat cortical neurons. J Neurosci 18:1935-1943.

Kiessling V, Muller B, Fromherz P (2000) Extracellular resistance in cell adhesion measured with a transistor probe. Langmuir 16:3517-3521.

Krapivinsky G, Krapivinsky L, Manasian Y, Ivanov A, Tyzio R, Pellegrino C, Ben-Ari Y, Clapham DE, Medina I (2003) The NMDA receptor is coupled to the ERK pathway by a direct interaction between NR2B and RasGRF1. Neuron 40:775-784.

Kullmann DM (1994) Amplitude fluctuations of dual-component EPSCs in hippocampal pyramidal cells: implications for long-term potentiation. Neuron 12:1111-1120

Kutsuwada T, Kashiwabuchi N, Mori H, Sakimura K, Kushiya E, Araki K, Meguro H, Masaki H, Kumanishi T, Arakawa M, Mishina M (1992) Molecular diversity of the NMDA receptor channel. Nature 358:36-41.

Lehre KP, Danbolt NC (1998) The number of glutamate transporter subtype molecules at glutamatergic synapses: chemical and stereological quantification in young adult rat brain. J Neurosci 18:8751-8757.

Lehre KP, Rusakov DA (2002) Asymmetry of glia near central synapses favors presynaptically directed glutamate escape. Biophys J 83:125-134.

Lester RAJ, Jahr CE (1992) NMDA channel behavior depends on agonist affinity. J Neurosci 12:635-643.

Lozovaya NA, Kopanitsa MV, Boychuk YA, Krishtal OA (1999) Enhancement of glutamate release uncovers spillover-mediated transmission by $\mathrm{N}$-methyl-D-aspartate receptors in the rat hippocampus. Neuroscience 91:1321-1330.

Lu HC, Gonzalez E, Crair MC (2001) Barrel cortex critical period plasticity is independent of changes in NMDA receptor subunit composition. Neuron 32:619-634.

Manabe T, Renner P, Nicoll RA (1992) Postsynaptic contribution to longterm potentiation revealed by the analysis of miniature synaptic currents. Nature 355:50-55.

Mathews GC, Diamond JS (2003) Neuronal glutamate uptake contributes to GABA synthesis and inhibitory synaptic strength. J Neurosci 23:2040-2048.

Min MY, Rusakov DA, Kullmann DM (1998) Activation of AMPA, kainate, and metabotropic receptors at hippocampal mossy fiber synapses: role of glutamate diffusion. Neuron 21:561-570.

Mitchell SJ, Silver RA (2000) Glutamate spillover suppresses inhibition by activating presynaptic mGluRs. Nature 404:498-502.

Momiyama A (2000) Distinct synaptic and extrasynaptic NMDA receptors identified in dorsal horn neurones of the adult rat spinal cord. J Physiol (Lond) 523:621-628.

Nusser Z, Lujan R, Laube G, Roberts JDB, Molnar E, Somogyi P (1998) Cell type and pathway dependence of synaptic AMPA receptor number and variability in the hippocampus. Neuron 21:545-559.

Oertner TG, Sabatini BL, Nimchinsky EA, Svoboda K (2002) Facilitation at single synapses probed with optical quantal analysis. Nat Neurosci 5:657-664.

Poncer JC, Malinow R (2001) Postsynaptic conversion of silent synapses during LTP affects synaptic gain and transmission dynamics. Nat Neurosci 4:989-996.

Prybylowski K, Fu ZY, Losi G, Hawkins LM, Luo JH, Chang K, Wenthold RJ, Vicini S (2002) Relationship between availability of NMDA receptor subunits and their expression at the synapse. J Neurosci 22:8902-8910.

Racca C, Stephenson FA, Streit P, Roberts JDB, Somogyi P (2000) NMDA receptor content of synapses in stratum radiatum of the hippocampal CA1 area. J Neurosci 20:2512-2522.

Rosenmund C, Clements JD, Westbrook GL (1993) Nonuniform probability of glutamate release at a hippocampal synapse. Science 262:754-757.

Rusakov DA (2001) The role of perisynaptic glial sheaths in glutamate spillover and extracellular $\mathrm{Ca}^{2+}$ depletion. Biophys J 81:1947-1959.

Rusakov DA, Kullmann DM (1998) Extrasynaptic glutamate diffusion in the hippocampus: ultrastructural constraints, uptake, and receptor activation. J Neurosci 18:3158-3170.

Rusakov DA, Harrison E, Stewart MG (1998) Synapses in hippocampus oc- 
cupy only $1-2 \%$ of cell membranes and are spaced less than half-micron apart: a quantitative ultrastructural analysis with discussion of physiological implications. Neuropharmacology 37:513-521.

Satake S, Saitow F, Yamada J, Konishi S (2000) Synaptic activation of AMPA receptors inhibits GABA release from cerebellar interneurons. Nat Neurosci 3:551-558.

Schikorski T, Stevens CF (1997) Quantitative ultrastructural analysis of hippocampal excitatory synapses. J Neurosci 17:5858-5867.

Schmitz D, Mellor J, Nicoll RA (2001) Presynaptic kainate receptor mediation of frequency facilitation at hippocampal mossy fiber synapses. Science 291:1972-1976.

Semyanov A, Kullmann DM (2000) Modulation of GABAergic signaling among interneurons by metabotropic glutamate receptors. Neuron 25:663-672.

Semyanov A, Kullmann DM (2001) Kainate receptor-dependent axonal depolarization and action potential initiation in interneurons. Nat Neurosci 4:718-723.

Steigerwald F, Schulz TW, Schenker LT, Kennedy MB, Seeburg PH, Kohr G (2000) C-terminal truncation of NR2A subunits impairs synaptic but not extrasynaptic localization of NMDA receptors. J Neurosci 20:4573-4581.

Stocca G, Vicini S (1998) Increased contribution of NR2A subunit to synaptic NMDA receptors in developing rat cortical neurons. J Physiol (Lond) 507:13-24.

Takumi Y, Ramirez-Leon V, Laake P, Rinvik E, Ottersen OP (1999) Differ- ent modes of expression of AMPA and NMDA receptors in hippocampa synapses. Nat Neurosci 2:618-624.

Tovar KR, Westbrook GL (1999) The incorporation of NMDA receptors with a distinct subunit composition at nascent hippocampal synapses in vitro. J Neurosci 19:4180-4188.

Trommald M, Jensen V, Andersen P (1995) Analysis of dendritic spines in rat CA1 pyramidal cells intracellularly filled with a fluorescent dye. J Comp Neurol 353:260-274.

Turrigiano GG (1999) Homeostatic plasticity in neuronal networks: the more things change, the more they stay the same. Trends Neurosci 22:221-227.

Ventura R, Harris KM (1999) Three-dimensional relationships between hippocampal synapses and astrocytes. J Neurosci 19:6897-6906.

Vogt KE, Nicoll RA (1999) Glutamate and gamma-aminobutyric acid mediate a heterosynaptic depression at mossy fiber synapses in the hippocampus. Proc Natl Acad Sci USA 96:1118-1122.

Williams K, Russell SL, Shen YM, Molinoff PB (1993) Developmental switch in the expression of NMDA receptors occurs in vivo and in vitro. Neuron 10:267-278.

Zampighi GA, Fisher RS (1997) Polyhedral protein cages encase synaptic vesicles and participate in their attachment to the active zone. J Struct Biol 119:347-359.

Zheng F, Erreger K, Low CM, Banke T, Lee CJ, Conn PJ, Traynelis SF (2001) Allosteric interaction between the amino terminal domain and the ligand binding domain of NR2A. Nat Neurosci 4:894-901. 